\title{
Identificación automática del canal de aprendizaje en mensajes cortos
}

\author{
Rocío Galaviz, Beatriz Beltrán, Andrés Vázquez, Mireya Tovar \\ Benemérita Universidad Autónoma de Puebla \\ Facultad de Ciencias de la Computación \\ Avenida San Claudio, 14 Sur, Ciudad Universitaria \\ Puebla, México \\ chio gus@hotmail.com, \{bbeltran, andrex, mtovar\}@cs.buap.mx \\ http://nlp.cs.buap.mx
}

\begin{abstract}
Resumen. En el presente trabajo se proponen varios modelos de clasificación para detectar el tipo de aprendizaje de un individuo a partir de los tweet que escribe. Este modelo se apoya en los tres tipos de canal de aprendizaje propuesto en la programación neurolingüística (visual, auditivo y kinestésico). 4 expertos desarrollaron un corpus de entrenamiento, clasificando de manera manual a 1000 tweets. Se desarrollaron diversos experimentos considerando diferentes modelos de representación y varios clasificadores. Se realizó validación cruzada de 10 pliegues y los mejores resultados fueron ofrecidos por el clasificador máquina de soporte vectorial.
\end{abstract}

Palabras clave: Canal de Aprendizaje, modelos de clasificación, representación por medio de $n$-gramas, Programación Neurolingüística.

\section{Introducción}

Durante años se ha trabajado para mejorar el nivel educativo en México, se han implementado diversas propuestas metodológicas para mejorar el proceso enseñanzaaprendizaje y los resultados obtenidos aun en la actualidad no son los esperados, sería conveniente que se ofrezcan herramientas automáticas que permitan detectar la forma en que los alumnos aprenden.

La Programación Neurolingüística (PNL) constituye un modelo, formal y dinámico de cómo funciona la mente y la percepción humana, cómo se procesa la información y la experiencia, y las diversas implicaciones que esto tiene para el éxito personal. Con base a este conocimiento es posible identificar las estrategias internas que utilizan las personas de éxito, aprenderlas y enseñarlas a otros (modelar); para facilitar un cambio evolutivo y positivo. La Programación Neurolingüística, por analogía con los sistemas de cómputo, utiliza los patrones universales de comunicación y percepción que tenemos para reconocer e intervenir en procesos diversos (aprendizaje, terapia, afrontamiento del estrés, negociación, gestión de conflictos, superación de fobias, etc.). El campo de trabajo es tan amplio como el de las relaciones interpersonales [1].

Dentro del área de la educación, los tipos de aprendizaje que la PNL maneja son 3: 
- Visual, son aquellos que comprenden y recuerdan más fácilmente la información si se les da por escrito. Para estudiar, a estas personas, les funciona muy bien hacer mapas conceptuales, buscar imágenes con que relacionar la información, escribir y leer apuntes.

- Auditivo, les es más fácil escuchar la información que leerla. Por lo general si toman apuntes se pierden, prefieren poner mucha atención a lo que escuchan y tienen una gran habilidad para recordar conversaciones. Su método de estudio puede implicar estudiar en voz alta, pedir a alguien que les explique de manera verbal o bien inventar rimas, canciones o juegos de palabras para memorizar.

- Kinestésico, aprenden a través del contacto directo con los contenidos y las emociones. Pueden fácilmente relacionar los contenidos con el estado de ánimo que les producen, estudian mejor en ambientes confortables y conocidos, muchos de ellos estudian mejor en compañía y optan por visitar museos y experimentar los contenidos [2].

En [6] se plantea que la PNL es un instrumento poderoso para reprogramar nuevamente la mente e introducirle información acerca de todos los principios que rigen una mala experiencia, para así desbloquear esas falsas creencias que se arraigaron en la mente y que no permiten tomar las acciones que conlleven a los resultados que deseamos.

La PNL parte de la teoría constructivista, que define la realidad como una invención y no como un descubrimiento. Se basa en el hecho de que el ser humano no opera directamente sobre el mundo real en que vive, sino que lo hace a través de representaciones mentales del mismo, que determinan la forma en que cada individuo percibe el mundo. Proporciona herramientas y habilidades para el desarrollo en comunicación y reprogramación de actitudes. Promueve la flexibilidad del comportamiento, la creatividad y la comunicación, el pensamiento trascendental y una comprensión de los procesos mentales, tanto para el desarrollo individual como para la optimización de cada grupo humano.

Existen diversos test en la web que normalmente manejan los psicólogos para detectar el canal de aprendizaje, sin embargo no son tan confiables, pues estos pueden ser alterados por los alumnos al responder. Es más conveniente detectar el canal de aprendizaje dentro de una plática común o incluso cuando el estudiante redacta algo que sale de su imaginación, como un cuento o la explicación de algún texto. Por lo que, se propone desarrollar una metodología que se pueda aplicarse al sector educativo y que le brinde al profesor las herramientas tecnológicas donde él pueda clasificar a los alumnos según su estilo de aprendizaje, así sabría cómo dirigirse de una forma más asertiva y qué métodos puede aplicar para mejorar el aprovechamiento del mismo.

La estructura del artículo, se tiene de la siguiente manera, en la sección 2, se presenta los fundamentos teóricos de la programación neurolingüística; en la sección 3, se presenta el trabajo que se ha realizado para la detección del canal de aprendizaje. En la sección 3 se describe la propuesta de metodología para la identificación del canal de aprendizaje dentro de textos cortos. En la sección 4 se muestran algunos resultados obtenidos y finalmente las conclusiones que se obtienen hasta el momento. 


\section{Trabajo Relacionado}

Dentro de la investigación realizada no se encontró ningún sistema que mediante el análisis de un texto de manera automática detecte el estilo de aprendizaje y que esté basado en la PNL, sin embargo se encontraron algunos sistemas con temas relacionados, los cuales se discuten a continuación:

Dentro del trabajo de tesis desarrollado en [3] se propone un sistema de apoyo para el aprendizaje de lecto-escritura para niños, su objetivo fue la realización de un módulo de dictado y corrección de ortografía, programado de una manera flexible para su integración a la arquitectura Galaxy como parte de un sistema tutorial de lectoescritura.

Por otro lado, el trabajo desarrollado en [4] se propone un sistema para el fortalecimiento del pensamiento matemático dirigido a niños de preescolar. El software propuesto se conforma de dos juegos que cubren distintas áreas del pensamiento matemático. Se utilizan los bloques mágicos para abordar principalmente el tema de número y el bloque denominado Circolar que se enfoca al tema de forma y figuras geométricas. Con la utilización del sistema en el proceso de enseñanza se pudo validar que los alumnos lograron de manera correcta apropiarse de los conceptos matemáticos involucrados.

Otra tendencia actual ha sido introducir las plataformas de aprendizaje en las diferentes materias que forman parte de la curricula a nivel básico y medio superior, sin embargo esto se ha visto limitado por las condiciones particulares de cada escuela. Otra tendencia ha sido el desarrollo de entornos virtuales de enseñanza-aprendizaje para la enseñanza en el nivel superior. En particular en [5] se desarrolló una plataforma de este tipo a través de la Web y que maneja 3 perspectivas:

- Perspectiva social y pedagógica, trabajando sobre aprendizaje colaborativo

- Perspectiva tecnológica, diseñar módulos para la plataforma educativa que permitan la configuración y la realización de experiencias con grupos de alumnos con tecnología basada en Internet y WWW.

- Perspectiva experimental, diseñar, configurar y realizar experiencias a distancia en entornos reales.

Como se comentó al inicio hasta el momento no se ha desarrollado ninguna herramienta tecnológica de apoyo al docente que le permita detectar de una manera adecuada la forma en que su grupo de trabajo aprende, en este sentido está encaminada la presente investigación.

A continuación se discute el modelo de clasificación desarrollado para detectar el canal de aprendizaje de un individuo.

\section{Fundamentos teóricos de PNL}

La PNL parte de los fundamentos de la teoría constructivista, la cual define la realidad como una invención y no como un descubrimiento. Es un constructo psíquico de Grinder y Bandler con base en el hecho de que el ser humano no opera directamente 
sobre el mundo real en el que se vive, sino que lo hace a través de mapas, representaciones, modelos a partir de los cuales se genera y guía su conducta. Estas representaciones, que además determinan el cómo se percibirá el mundo y qué elecciones se entenderán como disponibles, difieren necesariamente de la realidad de la cual representan. Esto se debe a que el ser humano, al transmitir su representación del mundo, tiene ciertas limitaciones, que se derivan de las condiciones neurológicas del individuo, de la situación social en que se vive y de sus características personales.

Estas limitaciones determinan que el lenguaje, como toda expresión humana, esté también sometido a ciertos procesos que empobrecen su modelo. Para el abordaje de estos modelos, la PNL se apoya en el modelo de la gramática transformacional que se basa en la creación de nuevos mensajes; considera que las personas son capaces de interpretar y producir mensajes nuevos, de manera que puede afirmarse que no se produce por repetición ni por recuerdo [7].

Se considera la PNL como:

- Una herramienta que ayuda a mejorar el aprendizaje

- Un esquema general práctico cuya finalidad es sernos útil, aumentar las opciones y conocernos mejor.

La PNL ayuda a entender como los seres humanos estructuramos las experiencias individuales de la vida; representa una actitud de la mente y una forma de ser en este mundo.

Desde 1976 los autores originales llaman PNL a su trabajo original [8]:

- Programación porque se refiere a un proceso organizador de componentes de un sistema para obtener un resultado esperado; a los modos que podemos escoger para organizar nuestras ideas (pensamientos-emociones) y acciones.

- Neuro porque se parte de todo comportamiento proviene de procesos del sistema nervioso (los sentidos y demás expresiones neurológicas) mediante los cuales hacemos contacto con el mundo y damos sentido a la información, actuando en consecuencia.

- Lingüística porque usamos el lenguaje para ordenar nuestras ideas y comunicarnos con los demás, como un recurso que tiene una representación, orden y secuencia que manifestamos.

La comunicación está formada por un conjunto de factores (gestos, posturas, palabras, tono de voz). Esta manera de comunicarnos viene determinada por nuestro mapa del mundo; es decir, nuestra manera de percibir y organizar la realidad. Y este proceso se da a través de nuestros sentidos. En la PNL las maneras como recogemos, almacenamos y codificamos la información en nuestra mente -ver, oír, gustar y oler- se conoce con el nombre de sistemas representativos o canales de aprendizaje:

- Visual (imágenes)

- Auditivo (sonidos, ritmo)

- Cinestésico o kinestésico (sensaciones, gusto y olfato) 
Cuando una persona tiende a utilizar, de manera habitual, un sentido de forma interna, se dice que éste es su sistema preferido o primario, el cual se refleja tanto en el vocabulario que utilizamos como en las posturas, tono de voz, gestos con los que acompañamos las palabras [7].

\section{Descripción de la propuesta}

Se confeccionó un corpus de entrenamiento conformado por 1,000 tweets considerando una muestra de 676 usuarios diferentes con un promedio de 1.479 tweets cada uno. Se desarrolló un diccionario de emoticones para normalizar los tweets, además cada usuario es sustituido por la etiqueta USER_TAG y los hash-tag por una etiqueta similar. Las características del corpus se muestran en la Tabla 1 y Tabla 2.

Tabla 1. Información del corpus de tweets

\begin{tabular}{cccccc}
\hline Tweets & $\begin{array}{c}\text { Mínimo de } \\
\text { palabras }\end{array}$ & $\begin{array}{c}\text { Máximo de } \\
\text { palabras }\end{array}$ & $\begin{array}{c}\text { Promedio de } \\
\text { palabras }\end{array}$ & Vocabulario & Tokens \\
\hline 1,000 & 1 & 32 & 11.757 & 4,215 & 11,757 \\
\hline
\end{tabular}

Tabla 2. Información de usuarios dentro del corpus

\begin{tabular}{cccc}
\hline $\begin{array}{c}\text { Mínimo de } \\
\text { tweets por } \\
\text { usuario }\end{array}$ & $\begin{array}{c}\text { Máximo de } \\
\text { tweets por } \\
\text { usuario }\end{array}$ & $\begin{array}{c}\text { Promedio de } \\
\text { tweets por } \\
\text { usuario }\end{array}$ & $\begin{array}{c}\text { Total de } \\
\text { usuarios }\end{array}$ \\
\hline 1 & 16 & 1.479 & 676 \\
\hline
\end{tabular}

En el proceso manual de clasificación se propone utilizar un total de 4 clases. La categoría neutro; es asignada si en el tweet no existen los elementos suficientes para identificar el canal de aprendizaje. La categoría de auditivo es asignada cuando a través del texto se detecta que aprende por medio de lo que escucha. La categoría visual es detectada si en el tweets se determina que su canal de aprendizaje es a través de lo que observa, por último si aprende por medio de lo que toca se dice que ese individuo es kinestésico. La cantidad de tweets por clase se muestra a continuación en la Tabla 3.

Tabla 3. Tweets por clase

\begin{tabular}{cr}
\hline Clase & Tweets por clase \\
\hline Neutro & 577 \\
Auditivo & 64 \\
Visual & 144 \\
Kinestésico & 267 \\
\hline
\end{tabular}


Tabla 4. Ejemplos de tweets de cada clase

\begin{tabular}{|c|c|}
\hline & $\begin{array}{l}\text { USER_TAG que tengas buen dia HAPPY_TAG } \\
\text { USER_TAG eee gracias * le da, una buena propina } \\
\text { I am at La Casa De Don Porfirio (Atlixco, Puebla) URL_TAG } \\
\text { Una niña vale más que veinte niños HAPPY_TAG } \\
\text { USER_TAG Visita la Capilla del Rosario en Recorrido Virtual HASH_TAG } \\
\text { URL_TAG }\end{array}$ \\
\hline$\stackrel{0}{:}$ & $\begin{array}{l}\text { Ya los acabo de escuchar, muy buenos USER_TAG USER_TAG } \\
\text { Por qué HOY? Por que HOY? ya estaba decidido a que mañana hablaría las } \\
\text { cosas por HOY pasa esto! PUTAAAAAAAMAAAADREEEEEE! La cagas } \\
\text { escucha una canción o reconoce en un desconocido un gesto, y todo se vuelve a } \\
\text { abrir, todo regresa, como una ola, como una bofetada } \\
\text { HASH_TAG Ella dijo que no le hable y no le hablare } \\
\text { Decir que un alto cociente intelectual no hace superior a nadie es aceptar que se } \\
\text { tiene uno bajo }\end{array}$ \\
\hline $\begin{array}{l}\bar{\pi} \\
\vec{\sigma} \\
\stackrel{7}{>}\end{array}$ & $\begin{array}{l}\text { algo no me cuadra... CONOCISTE A OTRA PERSONA? } \\
\text { RT USER_TAG El uso de zapatos blancos debería ser ilegal } \\
\text { Acabo de publicar una foto URL_TAG } \\
\text { Típico: hacer enojar a tu hermana, recordar que te puede acusar con tu mamá y } \\
\text { la haces en contentar } \\
\text { USER_TAG me deberías hacer así lindura }\end{array}$ \\
\hline 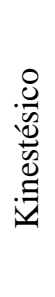 & $\begin{array}{l}\text { RT USER_TAG En domingo siempre me llega la nostalgia. URL_TAG } \\
\text { RT USER_TAG No es fácil olvidar a alguien que fue muy importante en tu vida } \\
\text { USER_TAG jaja de hecho no, expresé una disculpa y amablemente expresé mi } \\
\text { opinión sobre el tema. TONGUE-TAG } \\
\text { No tengo celos, pero tengo un rifle } \\
\text { Yo por ti dejo lo que tú me pidas yo te doy mi vida entera te la doy y te prometo } \\
\text { tu serás la reyna de mi fortaleza y de mi corazón }\end{array}$ \\
\hline
\end{tabular}

Con estos datos de entrenamiento se desarrollan varios modelos de clasificación. En particular se utilizan los clasificadores Naive-Bayes Multinomial, k-vecinos más cercanos (IBK), máquina de soporte vectorial (SMO) y árbol de decisión (J48), ofrecidos por la herramienta WEKA ${ }^{1}$.Se realizó validación cruzada con 10 pliegues, los resultados obtenidos por los 4 clasificadores se muestran en la siguiente sección.

\section{Resultados}

Para la representación de los tweets se utilizó un modelo de $n$-gramas, donde $n$ va del valor 1 hasta el valor 3 . Se desarrollaron varios experimentos considerando la frecuencia de cada $n$-grama en el rango de 1 a 3 . Los resultados obtenidos se muestran en la Tabla 5.

\footnotetext{
1 http://www.cs.waikato.ac.nz/ml/weka/
} 
Identificación automática del canal de aprendizaje en mensajes cortos

Tabla 5. Resultados utilizando diferentes clasificadores

\begin{tabular}{c|cccc|ccc}
\hline Clasificador & $\begin{array}{c}\text { Mín } \\
\text { frec }\end{array}$ & $\begin{array}{c}\boldsymbol{n} \text { - } \\
\text { gra- } \\
\text { mas }\end{array}$ & $\begin{array}{c}\text { Instancias } \\
\text { Correctas }\end{array}$ & $\begin{array}{c}\text { Instancias } \\
\text { Incorrectas }\end{array}$ & $\begin{array}{c}\boldsymbol{n} \text { - } \\
\text { gra- } \\
\text { mas }\end{array}$ & $\begin{array}{c}\text { Instancias } \\
\text { Correctas }\end{array}$ & $\begin{array}{c}\text { Instancias } \\
\text { Incorrectas }\end{array}$ \\
\hline $\begin{array}{c}\text { BAYES- } \\
\text { MULTINO- }\end{array}$ & 1 & $1-3$ & $\mathbf{6 0 . 9 3 1 6 \%}$ & $39.0684 \%$ & $2-3$ & $60.0760 \%$ & $39.9240 \%$ \\
$\begin{array}{c}\text { MIAL } \\
\text { IBK }\end{array}$ & 1 & $1-3$ & $59.3156 \%$ & $40.6844 \%$ & $2-3$ & $59.3156 \%$ & $40.6844 \%$ \\
SMO & 1 & $1-3$ & $60.3612 \%$ & $39.6388 \%$ & $2-3$ & $\mathbf{6 0 . 4 5 6 3 \%}$ & $39.5437 \%$ \\
J48 & 1 & $1-3$ & $55.6084 \%$ & $44.3916 \%$ & $2-3$ & $57.6996 \%$ & $42.3004 \%$ \\
\hline BAYES- & 2 & $1-3$ & $60.3612 \%$ & $39.6388 \%$ & $2-3$ & $57.4144 \%$ & $42.5856 \%$ \\
MULTINO- & & & & & & & \\
MIAL & 2 & $1-3$ & $55.038 \%$ & $44.962 \%$ & $2-3$ & $58.2700 \%$ & $41.7300 \%$ \\
IBK & 2 & $1-3$ & $\mathbf{6 1 . 0 2 6 6 \%}$ & $38.9734 \%$ & $2-3$ & $59.9810 \%$ & $40.0190 \%$ \\
SMO & 2 & $1-3$ & $56.0837 \%$ & $43.9163 \%$ & $2-3$ & $57.0342 \%$ & $42.9658 \%$ \\
\hline J48 & 3 & $1-3$ & $58.9354 \%$ & $41.0646 \%$ & $2-3$ & $56.3688 \%$ & $43.6312 \%$ \\
\hline BAYES- & & & & & & & \\
MULTINO- \\
MIAL
\end{tabular}

Los mejores resultados con un $61.0266 \%$ de instancias correctamente clasificadas, fueron obtenidos utilizando el clasificador máquina de soporte vectorial, donde tanto para los unigramas, los bigramas y los trigramas se tiene una frecuencia mínima de 2. La F1 muestra su valor máximo para la clase neutra, y su mínimo valor para la clase auditiva, eso se muestra en la Tabla 6. Este resultado se corresponde totalmente con las características del corpus de entrenamiento, ya que está totalmente desbalanceado.

Tabla 6. Precisión, evocación y F1 para el clasificador SMO

\begin{tabular}{cccccccc}
\hline & $\begin{array}{c}\text { TP } \\
\text { Rate }\end{array}$ & $\begin{array}{c}\text { FP } \\
\text { Rate }\end{array}$ & Precision & Recall & F1 & $\begin{array}{c}\text { ROC } \\
\text { Area }\end{array}$ & Class \\
\hline & 0.828 & 0.366 & 0.733 & 0.828 & 0.778 & 0.751 & NEUTRO \\
& 0.125 & 0.031 & 0.205 & 0.125 & 0.155 & 0.652 & AUDITIVO \\
& 0.25 & 0.087 & 0.313 & 0.25 & 0.278 & 0.61 & VISUAL \\
& 0.449 & 0.161 & 0.488 & 0.449 & 0.468 & 0.644 & KINESTESICO \\
\hline $\begin{array}{c}\text { Weighted } \\
\text { Avg. }\end{array}$ & 0.61 & 0.255 & 0.581 & 0.61 & 0.593 & 0.699 & \\
\hline
\end{tabular}

Los resultados obtenidos en la Tabla 7 muestran que la mayor cantidad de tweets clasificados incorrectamente, son aquellos que corresponden a tweets asociados a individuos con aprendizaje kinestésico. 
Rocío Galaviz, Beatriz Beltrán, Andrés Vázquez, Mireya Tovar

Tabla 7. Matriz de confusión para el clasficador SMO

\begin{tabular}{ccccccc}
\hline A & B & C & d & \multicolumn{2}{c}{ Clasificado como } \\
\hline 478 & 7 & 29 & 63 & a & $=$ & NEUTRO \\
25 & 8 & 11 & 20 & b $=$ & AUDITIVO \\
57 & 8 & 36 & 43 & c & $=$ & VISUAL \\
92 & 16 & 39 & 120 & d & $=$ & KINESTESICO \\
\hline
\end{tabular}

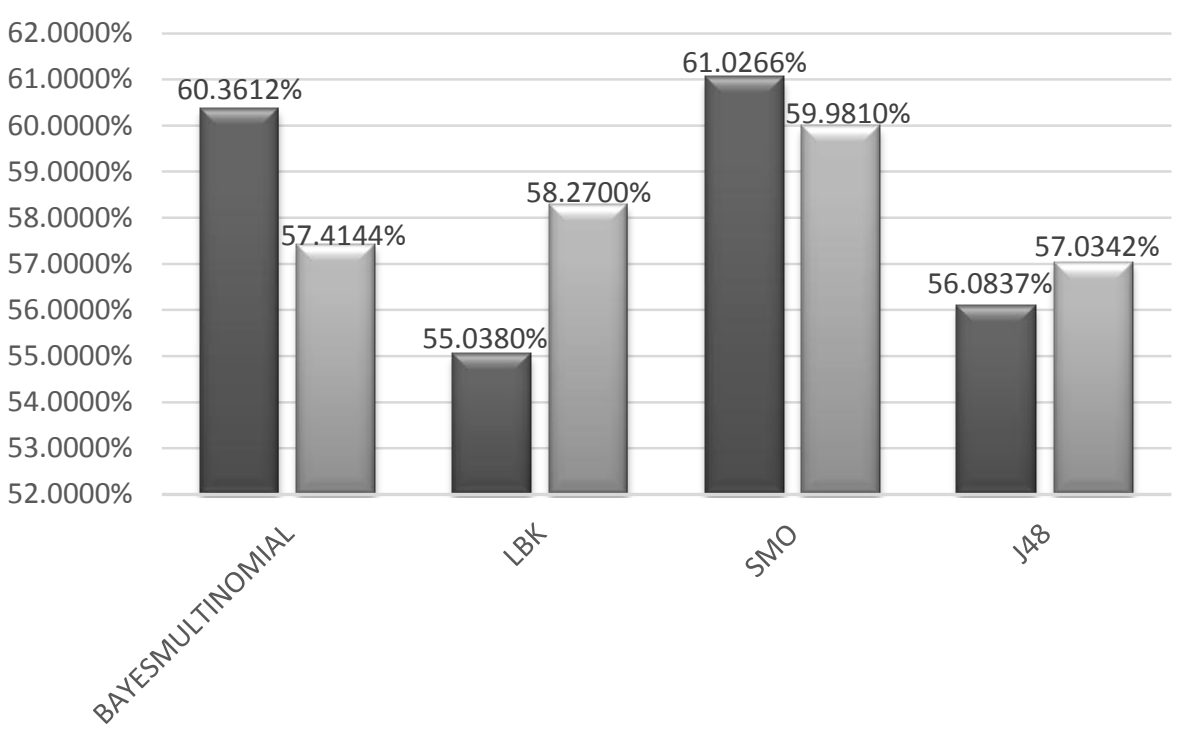

曰-3 $\square 2-3$

Fig. 1. Porcentajes para una mínima frecuencia de $2, n$-gramas de $1-3$ y de 2-3

En la Fig. 1 se muestran los porcentajes obtenidos por cada uno de los clasificadores, con una frecuencia mínima de 2 y para $n$-gramas de 1 a 3 y de 2 a 3 .

\section{Conclusiones}

Como resultado de este trabajo de investigación se ha desarrollado un corpus de entrenamiento en el que han participado 4 expertos en el área de programación neurolingüística, lo que nos permitirá proponer nuevos modelos de aprendizaje automático. Los resultados obtenidos son completamente lógicos, ya que el corpus no está balanceado, y se sabe que esto afecta en el proceso de clasificación. Se pudo comprobar que de los 4 clasificadores utilizados el mejor comportamiento lo ofreció la máquina de soporte vectorial utilizando bolsa de $n$-gramas, sin embargo se considera adecuado utilizar tf*idf. 
En estos momentos se está extendiendo el corpus de entrenamiento, buscando obtener tweets representativos de cada clase. Se están desarrollando modelos de entrenamientos considerando hasta quintigramas.

\section{Referencias}

1. Programación Neuroligüistica, http://es.wikipedia.org/wiki/Programación_neurolingüística (Visitada: 14/10/2014)

2. Canales del Aprendizaje, http://elpsicoasesor.com/canales-del-aprendizaje/ (Visitada: 14/10/2014)

3. Gutiérrez F.L.: Módulo de dictado para un sistema de apoyo para el aprendizaje de lectoescritura para niños. Tesis Universidad de las Américas Puebla. (2002)

4. Peralta H.I.: KidMatex: Software Educativo para el fortalecimiento del pensamiento matemático dirigido a niños de preescolar. Tesis Universidad de las Américas Puebla. (2010)

5. Gil, G., Arias, D., Sanchez, E., Gimson,L. Romero, R., Ramirez, J., E., Silvera, J., Massé, M., Fernández, E.: Desarrollo de un entorno virtual de enseñanza-aprendizaje para la Universidad Nacional de Salta. In XII Workshop de Investigadores en Ciencias de la Computación. WICC 2010. Santa Cruz, Argentina (2010)

6. Cuscó M., Solanich, R.: Actividades de programación neurolingüística para educación primaria. Aula de innovación educativa (2012)

7. Peterson D.: PNL conoce cómo te puede apoyar. Editores Emu Mexicanos Unidos, México (2013).

8. O’Connor, J. \& Seymour, J.: Introducción a la Programación Neurolingüística. Editorial Urano, Barcelona (1995) 